\title{
Looking for the Source of Change
}

\author{
L. S. Schulman',2 • M. G. E. da Luz ${ }^{3}$
}

Received: 26 February 2016 / Accepted: 23 June 2016 / Published online: 29 July 2016

(C) The Author(s) 2016. This article is published with open access at Springerlink.com

\begin{abstract}
In most theories of the quantum measurement process changes in an observer's perception of a state can take place without forces, as for example if a state is prepared in an eigenstate of $J_{x}$ ( $x$ component of angular momentum) but $J_{z}$ is measured. In the "special state" theory (explained in the previous article) any change in wave function requires forces. This allows experimental tests to distinguish these ideas and in the present article two examples of such tests are considered. The first is a kind of double Stern-Gerlach experiment, the second a check for angular momentum changes in a polarizer.
\end{abstract}

Keywords Special states - Arrow of time $\cdot$ Foundational experiments

\section{Introduction}

A companion article [1] includes an introduction to the special state theory of quantum measurement-basically that there is no such thing as a measurement, and there is only unitary time evolution [2]. In this the theory is similar to the many worlds interpretation, except that there is only one world. How this can be is explained there. The principal feature we focus on here is the fact that while most theories of the measurement

$凶$ L. S. Schulman

schulman@clarkson.edu

M. G. E. da Luz

luz@fisica.ufpr.br

1 Max Planck Institute for the Physics of Complex Systems, Nothnitzer Str 38, 10187 Dresden, Germany

2 Physics Department, Clarkson University, 5820, Potsdam, NY 13699, USA

3 Departamento de Física, Universidade Federal do Paraná, Curitiba, PR 81531-980, Brazil 
process allow force-free changes in the observer's perception of the value of certain observables, despite their being conserved, the special state theory does not.

The fact that both "Copenhagen" and "many worlds" allow changes in an observer's perception of an observable's value should come as no surprise. As an example, consider angular momentum. Let a Stern-Gerlach apparatus measure the $z$ component of angular momentum $\left(J_{z}\right)$ and send in an atom with its spin already oriented in (say) the $+z$ direction. There is no transfer or change in the world's total $J_{z}$ and in particular no transfer of $z$ component of angular momentum between atom and apparatus. The same is true if the spin is sent in oriented in the $-z$ direction. Now send in a superposition such that the orientation is (say) in the $x$ direction. By the superposition principal, there is still no transfer of angular momentum. However, to an observer there has been a change: from (the perception of) $J_{z}=0$ to $J_{z}$ being plus or minus $\hbar / 2$. (More details of this argument are given below and in [1].)

Section 2 of this paper considers details of a double Stern-Gerlach experiment in which the detection of an appropriate signal would support the special state theory over the alternatives. Following that, Sect. 3 considers whether a similar effect could be noted for photons, using polarization, or angular momentum, as the conserved quantity. The last section is a discussion.

\section{Angular Momentum in the Stern Experiment}

This involves a double Stern-Gerlach (SG) experiment, with something similar having been performed by Stern, Segrè, Frisch and Phipps in the early 1930s [3] ${ }^{1}$ in Stern's Hamburg laboratory. In our proposed setup one magnet prepares the spins (riding on $\mathrm{K}$ or Ag atoms) at some angle by appropriate orientation and by discarding half the output beam. A second magnet analyzes them along a different direction. The practical challenge in [3] was to create a region of zero magnetic field-otherwise the spins follow the field direction adiabatically.

The spin state, between magnets, can be taken to be

$$
u_{\theta}=e^{i \theta \sigma_{x} / 2}\left(\begin{array}{l}
1 \\
0
\end{array}\right)=\cos \frac{\theta}{2}|\uparrow\rangle+i \sin \frac{\theta}{2}|\downarrow\rangle, \quad \text { where } \sigma_{x}=\left(\begin{array}{ll}
0 & 1 \\
1 & 0
\end{array}\right)
$$

is one of the Pauli spin matrices and $\theta$ is a real number, related to the configurations of the two magnets. Other ways of arriving at the state $u_{\theta}$ are certainly possible, for example Bohm's version of the Einstein-Podolsky-Rosen experiment [4]. ${ }^{2}$ For definiteness though, we take the preparation to have occurred by means of a second Stern-Gerlach setup. Also, for simplicity at this stage, we assume that the state in Eq. (1) really is

\footnotetext{
1 This article summarized previous work in which at first Phipps and Stern did not succeed [20] but later Frisch and Segrè did [21]. The latter also relied on theoretical work of Majorana [22] in the analysis of the experiment. Translations of [21] and [22] are available from LSS although they should not be relied upon (the translator is not fluent in either language and relied on Google Translate for much of the task).

2 A spin-0 system decays to two spin-1/2 particles and the measurement of one of these shows it to be spin-up (in the $x$ direction). A measurement of the second particle made in another direction can be used to define a $z$-axis.
} 
the state-this will be examined later. In conventional quantum mechanics no force is required for a later reading of either spin up $(|\uparrow\rangle)$ or down $(|\downarrow\rangle),{ }^{3}$ where we assume the second magnet sorts the spins according to their $z$-axis orientation. For example, in the many worlds interpretation (MWI) an observation of spin-up would lead that (copy of the) observer to say, "Oh, I guess I'm on the $\cos (\theta / 2)|\uparrow\rangle$ branch."

On the other hand, in the special state theory the wave function had to evolve under unitary dynamics to the spin-up state (or spin-down, as the case may be), meaning there must be some Hamiltonian such that $\exp (-i H t / \hbar)$ takes it there. Bear in mind that the Hamiltonian involves far more degrees of freedom than the spin alone. Moreover, at the end of the experiment there is only one observer and no entanglement.

As emphasized in [1], among the rare states that might accomplish this one should choose the least rare. To find such a state some familiarity with the experiment is necessary. What needs to be accomplished is to have all atoms end in one of two places, the targets for spin-up and spin-down on the final detector. This can be done in a number of ways. What we think happens is that a magnetic field acts on the spin before it enters the second magnet and rotates it to an up or down configuration. But other possibilities exist and we want to argue that they are less likely than that suggested.

Another scenario that might seem likely is a force that acts after the atom emerges from the second magnet. At that point the wave function has divided into two portions and if one of them is acted on by a force it could be sent to the target region of the other. Since the final detector does not measure spin, but only location, this would seem to accomplish what is needed. A rough calculation (based on typical experimental conditions - about which more later) shows that at the exit from the second magnet the separation of the two portions of the wave function would be about $1 \mathrm{~mm}$, big by atomic standards. It would be traveling in the transverse direction at about $7 \mathrm{~m} / \mathrm{s}$ (in its principal direction the velocity is about $500 \mathrm{~m} / \mathrm{s}$ ). If the force acts for a microsecond, its strength should be about $10^{-19} \mathrm{~N}$. That such a deflection is possible seems reasonable-after all, scattering by a single (other) atom could deflect it in pretty much any direction. But this idea fails entirely because of entanglement. Whatever acts on this portion of the wave function should not act on the other, since that is already traveling in the right direction. This means that the degrees of freedom that apply the force to this branch of the wave function are now entangled with those of the spin-bearing atom (see [5] for similar issues in detection). For the special state theory residual entanglement is forbidden. More complex interactions in which the the same degrees of freedom act differently on the two branches of the wave function, which then separate unentangled, seem yet more unlikely.

One could also imagine that the forces within the magnet are somehow changed. But those forces are large, with the principal field about half a tesla and the gradient more than 1 tesla per $\mathrm{cm}$. By comparison-as we shall see-much smaller fields are needed to rotate the spin before it enters the strong field.

We therefore turn to the idea that a short term magnetic force acts to bring $u_{\theta}$ to $|\uparrow\rangle$ or $|\downarrow\rangle$ (up to phase factors). The first point to investigate is how large a field is needed. Unfortunately we do not know much about the special state that does this rotating;

3 By "force" we mean force that affects angular momentum. For both up and down spin orientations there will be very small, but different, forces in the $z$ direction, conserving linear momentum. 
presumably it involves quite a few degrees of freedom. However, we do know that in the end it must affect the spin through its interaction Hamiltonian which is simply $-\boldsymbol{\mu} \cdot \boldsymbol{B}$. What will be demanded of the field is that it causes the angle in Eq. (1) to rotate by an angle $\phi=-\theta+n \pi$ (with $n$ an integer) in order bring the spin entirely to an up or down state. Therefore we require that $\phi$ be at least of order unity, i.e.,

$$
|\phi|=\left|\frac{-\boldsymbol{\mu} \cdot \boldsymbol{B} \Delta t}{\hbar}\right|=\mathrm{O}(1),
$$

where $\Delta t$ is the time during which the special state acts. This assumes that $\boldsymbol{B}$ is roughly constant during its action. From the known values of $\hbar$ and $\boldsymbol{\mu}$ this requires that

$$
B \Delta t \geq 10^{-11} \text { Ts } .
$$

This is a lower bound. If, as argued in [1], the $\phi$ 's are Cauchy distributed then the distribution has neither a first nor a second moment. This implies that occasionally there will be large values of $n$ (hence $B$ ) occurring, making detection easier. In an earlier article [6] the expectation value for the angles involved in the kick are computed, but the sums involved only exist when matching positive and negative kicks ( $n$ 's) are added. ${ }^{4}$ As a result large fluctuations are expected.

Without knowing the source of the $\boldsymbol{B}$ field (in Eq. (3)) it is difficult to estimate $\Delta t$, but whatever it is, it must not be longer than the traversal time from one (SG) setup to the next. The distance scale is centimeters (call it $L$ and take $L=10 \mathrm{~cm}$ ) and velocities are about $500 \mathrm{~m} / \mathrm{s},{ }^{5}$ so that $\Delta t \leq 2 \times 10^{-4} \mathrm{~s}$. Using Eq. (3) this implies that $\boldsymbol{B}$ must be at least $5 \times 10^{-8}$ T or about 0.0005 gauss. ${ }^{6}$ This is about one thousandth the earth's field, but is well within the range of measurement. Presumably this field is also time-dependent, so that by Maxwell's equations there is necessarily an electric field. From $\nabla \times E=-\partial B / \partial t$ we make the rough estimate $E \sim B L / \Delta t \sim B v \sim$ $2.5 \times 10^{-5} \mathrm{~V} / \mathrm{m}$.

A number of practical issues enter and it is not clear whether any of them presents an insurmountable problem. Nevertheless, in the interest of full disclosure, We list anticipated problems.

1. Vacuum. This measurement must be done in a vacuum. Molecular beams require a good vacuum $\left(10^{-6}\right.$ Torr in the references mentioned in footnote 5$)$.

2. Zero field along the way. In Stern's double SG experiment [3] there was considerable difficulty in creating a region of zero magnetic field, necessary to avoid having the spins adiabatically follow the magnetic field.

\footnotetext{
${ }^{4}$ The sum in evaluating the expectation is not absolutely convergent. It is of the form $\sum_{n=-N_{1}}^{N_{2}} n /\left(1+n^{2}\right)$. So long as $N_{1}=N_{2}$ the answer is zero, but otherwise it can be any real number.

5 The numerical estimates in our article are based on information in references [23-25]. There are differences among them. References $[23,24]$ are for contemporary students reproducing the experiment and generally use $\mathrm{K}$ atoms and lower temperatures. On the other hand, $[25,26]$ tell the story of the original Stern-Gerlach experiment, which involved $\mathrm{Ag}$ atoms.

6 This corrects an error in [6] where a shorter time, hence larger field, was given. LSS thanks Amos Ori of the Technion for pointing this out to him.
} 
3. Fluctuations in the field near the magnets. The fields along the principal direction in the SG experiment is about $1 / 2 \mathrm{~T}$, and the gradient about $1 / 6 \mathrm{~T} / \mathrm{mm}$. With such strong fields you would need considerable stability to distinguish the relatively small fields that provide the kicks. This is especially true for the regions where the atom enters and exits the magnets, where considerable variation is expected [6].

4. Looking for the kick along the whole path. Using Eq. (1) we assumed a particular orientation for the spin. But the only way of knowing this is the preparation process itself. Therefore one must look along the entire path of the atom, from the exit from one magnet, to the entry of the other.

5. Perhaps the magnetic field does its rotating after the atom enters the strong magnetic field. This too seems unlikely because it would demand a complicated choreography involving both spin and translational degrees of freedom-once in the field, its gradient tends to separate the differing spin components.

\section{Angular Momentum for Photons}

Consider an experiment with photons, using the relation of polarization to angular momentum. Send a single circularly polarized photon through a birefringent crystal. For definiteness, let the direction of motion be $\hat{z}$ and the plane of incidence the $y-z$ plane. Let $\hat{u} \equiv(\hat{x}+\hat{y}) / \sqrt{2}$ and $\hat{v} \equiv(\hat{x}-\hat{y}) / \sqrt{2}$. Then up to constants the electric field of the photon at some time prior to its incidence on the crystal can be described by $E_{0} \phi(\mathbf{r})(\hat{u}+i \hat{v})$, with $\phi$ a function of position. The photon thus has helicity 1 , i.e., angular momentum $\hbar$ along the $z$-axis. Now let it pass through a beam splitter (e.g., calcite) that separates it into S and P polarizations, in this case along the $\hat{x}$ and $\hat{y}$ directions, respectively. Each of these linearly polarized states has zero helicity along the $z$-axis. If necessary, the exit angle of the P polarized field can be adjusted so the direction of travel is collinear with the original source of the photon-this to avoid issues of orbital angular momentum. Now someone measures this split photon and finds it in one of two locations - the beam splitter breaks the photon into two separated wave packets, as was the case for the Stern-Gerlach setup.

Conventionally, however, as for the Stern-Gerlach setup, the observer need not see any force for this change. From the Copenhagen standpoint, since $z$-axis helicity was not measured it was not defined. Of course it was prepared in a helicity state, but that does not matter: $J_{z}$ does not commute with the observable being measured. From the MWI perspective we could again analyze the what would happen if linearly polarized photons had been put through and then for the helicity state, after the measurement, each (copy of the) observer would attribute the outcome to having been on the appropriate branch.

For the special state theory these explanations do not work and to change the wave function something has to act. The questions are, what is the least unlikely way for this to happen and how to detect the appropriate force.

In general, changes in helicity are best accomplished through the collective action of many atoms or molecules arranged in repeating patterns, as in a crystal. Assuming that the special state is a cooperative effect of this sort, the mechanism would be some unusual movement taking place within (say) the birefringent crystal. Given current efforts at miniaturizing such devices the experiment may well be feasible. 
Microscopic birefringent crystals are now made [7] and transfers of tiny amounts of angular momentum are being measured [8,9]. In fact, since the work of Beth [10] showing that light's angular momentum could be translated into mechanical energy, there has been a remarkable level of miniaturization as well as applications [11-15]. Moreover, the mechanism of this detection, the torsion pendulum, is itself the subject of recent activity, especially in connection with gravitational wave detectors [16-19].

The form of detection that we have in mind would be the observation of the change in the polarizer's angular momentum. Since (in the special state theory) there is only one world, a change in the photon's angular momentum would have to be matched by a change in the polarizing device. By coupling the miniature polarizer to a sensitive torsion pendulum such a change could be detectable. There would be competing sources of rotation, including thermal noise as analyzed in several of the papers cited. While this experiment seems less feasible than the Stern-Gerlach test, the fact that so much work is now being done with photons suggests that it might be attempted.

One factor that should be useful is the boson character of the photons. If one could place a large number of them in the same state, the corresponding change in angular momentum would be that much greater.

\section{Discussion}

In the special state explanation for the definiteness of quantum measurement there is only unitary time evolution and only one world. This has experimental implications. In particular, if the observer - the only version of this observer that there is-prepares a state in an eigenstate of one operator and finds it in an eigenstate of a non-commuting operator, then there must be a Hamiltonian that acts on the wave function to make the change. (This is not necessary in the MWI interpretation, nor, as far as we know, in the various versions of the Copenhagen interpretation.)

In this article we have given two examples of the implementation of this idea, both involving angular momentum. In both cases it was necessary to find the least unlikely way to accomplish this change. For the Stern-Gerlach variation the signal strength of the special state seemed larger, but for the photon polarization example, the fact that minuscule photon effects are now being actively studied suggested that this test could more easily be implemented.

Finally, it must be said that we are realistic about the possibility that some version of this test will take place. Experimentalists are generally under funding pressure and long-shot experiments, whatever the payoff of success, can ill be afforded. Still it's remarkable how many fundamental experiments do take place, so perhaps optimism is warranted

Acknowledgments LSS is grateful to Amos Ori of the Technion for helpful discussions. The authors thank the Brazilian program Ciência Sem Fronteiras-CNPq (project 402193/2012-1) for financial support. Open access funding has been provided by the Max Planck Society.

Open Access This article is distributed under the terms of the Creative Commons Attribution 4.0 International License (http://creativecommons.org/licenses/by/4.0/), which permits unrestricted use, distribution, and reproduction in any medium, provided you give appropriate credit to the original author(s) and the source, provide a link to the Creative Commons license, and indicate if changes were made. 


\section{References}

1. Schulman, L.S.: Special states demand a force for the observer. Found. Phys. (2016). doi:10.1007/ s10701-016-0025-8

2. Schulman, L.S.: Time's Arrows and Quantum Measurement. Cambridge University Press, New York (1997)

3. Frisch, R., Phipps, T.E., Segrè, E., Stern, O.: Process of Space Quantisation. Nature 130, 892-893 (1932)

4. Bohm, D.: Quantum Theory (Dover, Mineola, NY, 1989), originally published by Prentice-Hall, Englewood Cliffs (1951)

5. Schulman, L.S.: Destruction of interference by entanglement. Phys. Lett. A 211, 75-81 (1996)

6. Schulman, L.S.: Experimental test of the "Special State" theory of quantum measurement. Entropy 14, 665-686 (2012)

7. Arita, Y., Mazilu, M., Dholakia, K.: Laser-induced rotation and cooling of a microgyroscope in vacuum. Nat. Commun. 4, 2374 (2013)

8. Kolesov, R., Xia, K., Reuter, R., Jamali, M., Stöhr, R., Inal, T., Siyushev, P., Wrachtrup, J.: Mapping spin coherence of a single rare-earth ion in a crystal onto a single photon polarization state. Phys. Rev. Lett. 111, 120502 (2013)

9. Rothmayer, M., Tierney, D., Frins, E., Dultz, W., Schmitzer, H.: Irregular spin angular momentum transfer from light to small birefringent particles. Phys. Rev. A 80, 043801 (2009)

10. Beth, R.A.: Mechanical detection and measurement of the angular momentum of light. Phys. Rev. 50, 115-125 (1936)

11. Chen, J., Ng, J., Ding, K., Fung, K.H., Lin, Z., Chan, C.T.: Negative optical torque. Sci. Rep. 4, 6386 (2014)

12. Hakobyan, D., Brasselet, E.: Optical torque reversal and spin-orbit rotational Doppler shift experiments. Opt. Exp. 23, 31230-31239 (2015)

13. Hernandez, R.J., Mazzulla, A., Provenzano, C., Pagliusi, P., Clipparrone, G.: Chiral resolution of spin angular momentum in linearly polarized and unpolarized light. Sci. Rep. 5, 16926 (2015)

14. Liu, H.: Optical spanner based on the transfer of spin angular momentum of light in semiconductors. Opt. Commun. 342, 125 (2015)

15. O'Neil, A.T., MacVicar, I., Allen, L., Padgett, M.J.: Intrinsic and extrinsic nature of the orbital angular momentum of a light beam. Phys. Rev. Lett. 88, 053601 (2002)

16. Bantel, M.K., Newman, R.D.: High precision measurement of torsion fiber internal friction at cryogenic temperatures. J. Alloys Compd. 310, 233-242 (2000)

17. Carbone, L., Cavalleri, A., Dolesi, R., Hoyle, C.D., Hueller, M., Vitale, S., Weber, W.J.: Upper limits on stray force noise for LISA (2013). arXiv:gr-qc/0310119 v1 (2003)

18. Pollack, S.E., Turner, M.D., Schlamminger, S., Hagedorn, C.A., Gundlach, J.H.: Charge management for gravitational-wave observatories using UV LEDs. Phys. Rev. D 81, 021101(R) (2010)

19. Schlamminger, S., Hagedorn, C.A., Famulare, M.G., Pollack, S.E., Gundlach, J.H.: High sensitivity torsion balance tests for LISA proof mass modeling. arXiv:gr-qc/0702053 v1 (2007)

20. Phipps, T.E., Stern, O.: Uber die Einstellung der Richtungsquantelung. Z. Phys. 73, 185-191 (1932)

21. Frisch, R., Segre, E.: Über die Einstellung der Richtungsquantelung. II. Z. Phys. 80, 610-616 (1933). English title: On the Process of space quantization. II. A translation is available (but see footnote 1 for a disclaimer) from LSS on request

22. Majorana, E.: Atomi orientati in campo magnetico variabile. Nuov. Cim. 2, 43-50 (1932). English title: Atoms in an oriented, variable magnetic field. A translation is available (but see footnote 1 for a disclaimer) from LSS on request

23. MIT Dept. Physics, Junior lab. The Stern-Gerlach Experiment: Quantization of Angular Momentum (2003). URL:web.mit.edu/8.13/JLExperiments/JLExp_18_rev1.pdf

24. Stern-Gerlach Experiment: catalogue description of the Stern-Gerlach experiment along with information about the experiment itself. PHYWE Systeme, GMBH, Göttingen, Germany (2011)

25. Friedrich, B., Herschbach, D.: Stern and Gerlach: how a bad cigar helped reorient atomic physics. Phys. Today. 53-59 (2003)

26. Bernstein, J.: The Stern Gerlach experiment (2010). arXiv:1007.2435 [physics.hist-ph] 